\title{
Phonon Scattering in Graphite
}

by

Paul Wagner 
Printed in the United States of America. Available from National Technical Information Service

U.S. Department of Commerce 5285 Porz Royal Road Springfield, VA 22161

Price: Printed Copy \$3.50 Microfiche \$2.25

Thin regort wal prepared ak en eccoust of work mpepeored by the United Silalem Government. Neither the Uniled Rtales

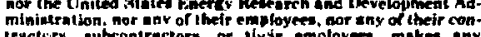

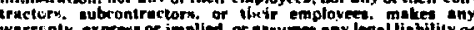

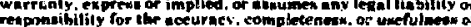

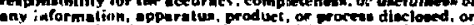

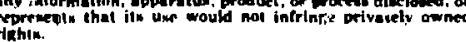


PHONON SCATTERING IN GRAPHITE

by

Paul Wagner

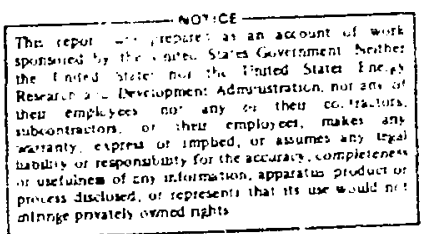

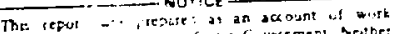

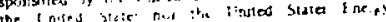

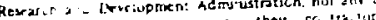

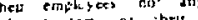

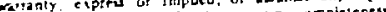

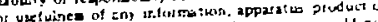

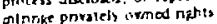

\section{ABSTRACT}

Effects on graphite thermal conductivities due to controlied alterations of the graphite structure by impurity addition, porosity, and neutron irradiation are shown to be consistent with the phenon-scattering formulation

$$
1 / \ell=\sum_{i=1}^{n} 1 / \ell_{i} .
$$

Observed temperature effects on these doped and irradiated graphites are also explained by this mechanism.

\section{INTRODUCTION}

A number of investigators ${ }^{1-8}$ have had varying degrees of success in trying to analyze, in whole or in part, the mechanism of thermal conduction in graphites. Application of theory to experiment has been even less successful. One major reason has been use of data from experiments in which the variables were poorly controlled or in which extraneous variables were allowed to interfere with the obser. vations. This report discusses, in terms of a simple phonon-scattering mechanism, the effects of carefully controlled impurity and defect additions, porosity, and temperature on measured thermal conductivities. There is strong evidence that graphite thermal conductivity behavior is consistent with this mechanism.

\section{THEORY}

The two major mechanisms by which heat is conducted in graphite are electronic motion and excitation of the acoustic modes in the graphite lattice. To a very good approximation, these mechanisms are independent and the total thermal conductivity, $\lambda$, may be written as the sum of the components.

$\lambda=\lambda_{E}+\lambda_{L}$.

$\lambda_{t}$ is commonly evaluated for metal systems by

$\lambda_{E}=L \sigma T$

(Weideman-Franz law), where $T$ is the temperature; $c$, the electrical conductivity; and $\mathbf{L}$, the Lorentz function whose limiting value is $2.45 \times 10^{-8}$ (V/degree) ${ }^{2}$ für fully degenerate systems. Separation of the two thermal conductivity components for semiconductors is a little more involved. However, it may be done by altering the electrical conductivity (usually by doping the matrix material with small amounts of impurities that change the electron or hole concentration) and measuring $\lambda$ as a function of $\sigma$. Since

$\lambda=\lambda_{2}+\operatorname{LoT}$,

a plot of $\lambda$ vs $\sigma$ extrapolated to $\sigma=0$ permits uetermination of $\lambda_{L}$. This is, of course, true only for those 
rare systems in which the impurity addition does not affect $\lambda_{L}$. So far, efforts to distinguish between $\lambda_{L}$ and $\lambda$ in graphite by these ard other methods have not been very successful.4,9-12 Here, this particular aspect of the problem is not treated. Instead, $\lambda_{L}$ is considered without reference to $\lambda_{t}$, and, in this instance, the generality of the results seems unaffected by the omission. For graphite, the total observed thermal conductivity is given by Eq. (1); however, at room temperature $(300 \mathrm{~K}) \lambda_{\mathrm{L}}>\lambda_{\mathrm{e}}$ and the expreasion

$\lambda=\lambda_{L}$,

although not exact, is a very reasonable approximation, as may be demonstrated by the following. Typical approximate values of $\lambda$ and $\sigma$ for a polycrystalline graphite at $300 \mathrm{~K}$ are about 150 $\mathrm{W} / \mathrm{m}-\mathrm{K}$ and $10^{3} \mathrm{mho} \mathrm{cm}^{-1}$, respectively. By Eq. (2), $\lambda_{t} \approx 0.735 \mathrm{~W} / \mathrm{m}-\mathrm{K}$ and $\lambda_{t} / \lambda_{\mathrm{L}} \approx 0.005$.

The lattice contribution to the thermal conductivity is limited by the various primary phonon scattering processen th: $x$ can occur at crystallite boundaries, at defect or impurity sites of almost any kind, or Es a result of phonon-phonon interaction (Umklapp processes). Generally, if a single mean free path, $\ell$, can be defined for each class of scatterer, it is proper to take the sum of the scattering probabilities to define the resultant mean path* as 6,13

$\frac{1}{l}=\sum_{1=1}^{n} \frac{1}{l_{1}}$.

or, more specifically,

$\frac{1}{l}=\frac{1}{l_{B}}+\frac{1}{l_{U}}+\frac{1}{l_{D}}$,

where

$\hbar_{B}=$ mean distance between crystallite boundaries,

$\varepsilon_{v}=$ mean free path for phonon-phonon scattering,

$G=$ mean free peth for (phonon) scattering by defects or impurities.

If the defect concentration is changed, the mean free path for defect scattering changes, ${ }_{D} \rightarrow l_{D}^{\prime} f \rightarrow l^{\prime}$, and $\mathrm{Eq}$. (6) becomes

*This is, in essence, Mattheissen's rule as applied to thermal resistance.
$\frac{1}{l^{\prime}}-\frac{1}{l_{B}}+\frac{1}{l_{V}}+\frac{1}{l_{D}^{\prime}}$.

If $\beta_{B}$ and $\ell_{1}$ are assumed to have remained constant, the ratio of esultant mean free paths may be determined by dividing Eq. (6) by Eq. (7) to get

$$
\left(\frac{l^{\prime}}{l}-1\right)\left(\frac{l_{D}}{l_{U}}+\frac{l_{D}}{l_{B}}\right)+\frac{l^{\prime}}{l} \frac{l_{D}}{\frac{q_{D}^{\prime}}{d}}-1=0
$$

and

$\frac{l^{\prime}}{l}-\frac{1+A}{\frac{l_{D}}{l_{D}^{\prime}}+A}$,

where

$A-\left(\frac{l_{D}}{l_{U}}+\frac{l_{D}}{l_{B}}\right)$.

As the test of the theory requires experiments in which only $b$, and thus $\&$ may change, we must examine the validity of the assumptions about the constancy of $l_{B}$ and $\ell_{U}$. Graphite has a hexagonal crystal structure with an extended $a-b$ planar ring structure, and a 1.42-A C.C bond length. The layer plane stacking is of the ABAB type with interplanar $c$-axis distances of $3.35 \AA$ or more, depending on the degree of crystallinity of the graphite. The forces acting between the atoms in the $a-b$ plane are due to strong covalent bonds, whereas those acting between the layer planes are of the weaker Van der Waals type. Because of this anisotropy in the structure, in. plane phonon processes are treated under the assumption that the layer planes are uncoupled.1,14 In fact, c-direction contributions can be ignored because of this uncoupling that permits one to consider only those processes that affect the properties in the in-plane direction, as is done here. Graphites are basically polycrystalline, and the mean distance between crystallite boundaries in the $a-b$ direction corresponds to $/ \mathrm{B}$ (equal to the crystallographic $\mathrm{L}_{\mathrm{a}}$ ). This distance is a measurable crystallographic parameter, and the constancy of $l_{B}$ may be ensured either by measurement (in going from one test specimen to another) or by changes induced in a single test sample, as by irradiation which can change the defect concentration without necessarily altering crystallite size. 
The validity of the assumption that $f_{U}$ is constant cannot be established, so the concept expressed by Eq. (7) must be abandoned. The reasoning is as follows; past work on how irradiaticn influences the thermal conductivities of polycrystalline graphites $^{11,15,16}$ and how boron impirity addition influences them 12 shows that near $300 \mathrm{~K}$ the total $\lambda$ decreases with increased defect or impurity concentration. Irradiation decreases $\sigma$ (electrical conductivity), whereas boron doping increases it. Thus the observed effect is clearly due to changes in $\lambda_{\mathrm{L}}$ and falls within the scope of this work. Since the thermal conductivity lattice component is related to the mean free path by the Debye relationship

$$
\lambda_{L}=\frac{1}{3} \sum_{1=1}^{n} c_{1} v_{i} \ell,
$$

where

$\mathrm{C}=$ heat capacity,

$v=$ phonon velocity,

$\mathrm{i}=$ scoustic node index,

and $/$ is given by Eq. (6), the observed effect on the thermal conductivity must be due to a lack of constancy in $t_{U}$ or $l_{D}$ or both, assuming that the constancy of ${ }_{B}$ has been established. Work at this Laboratory on effects of silicon doping in polycrystalline graphite, to concentration levels comparable with the boron work cited, showed essentially no change in $\lambda$ or $\sigma$ (see Table I). In this instance, a change in defect concentration affected neither $t_{\mathrm{O}}$ nor $b\left(t_{\mathrm{B}}\right.$ was constant $)$. Therefore, it appears that the constency of $\ell_{U}$ or $\ell_{D}$ as a function of defect concentration cannot he established a priori and, furthermore, that the two may not be separable. Probably the major ultimate effect of changing the defect concentration and thus introducing new scattering centers is altered periodicity of the potential field associated with the graphite structure which alters the valu of $/ U$. Perhaps it is more reasonable to rewrite $\mathrm{Eq}$. (6) as

$$
\frac{1}{\ell}=\frac{l}{l_{B}}+\frac{l}{l_{U}},
$$

where $f_{U}$ is understood to contain defect and impurity effects and where $f_{\mathrm{y}} \rightarrow f_{\mathrm{U}}^{\prime}$ as the defect concentrution is changed. As in Eqs. (7)-(9),

\section{TABLE I}

\section{INFLUENCE OF SILICON ADDITION ON ELECTRICAL AND THERMAL PROPERTIES OF POLYCRYSTALLINE GRAPHITES}
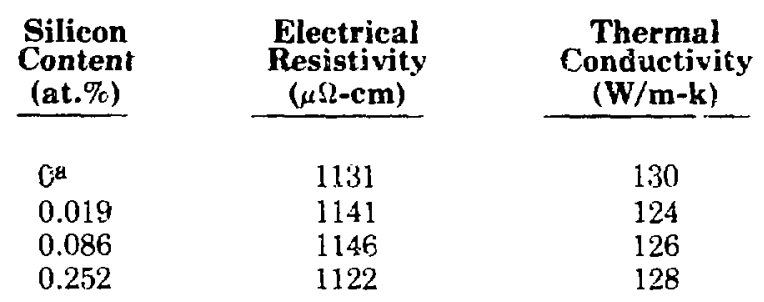

a Pure graphite.

$$
\frac{\ell^{\prime}}{\ell}=\frac{\frac{1}{\ell}+F}{\frac{U}{\ell_{U}^{\prime}}+F},
$$

where

$$
P=\frac{\ell_{U}}{l_{B}} \text {. }
$$

The similarity between Eqs. (9) and (13) is obvious and seems to be quite general for any number of variables treated as is done here. In other words, whether Eq. (6) contains two terms or $n$ terms is immaterial, inasmuch as the final working equation is of tne same form, and only the terms in the constant are different. Since $i_{\mathrm{U}}^{i}$ snould decrease with increased defect concentration, $\ell_{U} / \ell_{U}^{\prime}$ should be proportional to impurity concentration, total radiation dosage, or any other variable that increases defect concentration. Also, the observed thermal conductivity will be directly proportional to $\prime^{\prime}, \mathrm{Eq}$. (11), so a graph of $\lambda$ vs defect concentration or exposure to radiation is similar to a plot of $\ell^{\prime} / / \mathrm{vs} f_{\mathrm{L}} / /_{\mathrm{L}}^{\prime}$. Differentiating Eq. (13) gives

$$
\frac{d\left(\ell^{\prime} / l\right)}{d\left(\ell_{U^{\prime}} / \ell_{v}^{\prime}\right)}=-\frac{(1+F)^{2}}{\left(\frac{\ell_{U}}{\ell_{U}^{\prime}}+F\right)^{2}} .
$$

Since $f_{U} / f_{U}^{\prime}>0$, a plot of $f^{\prime} f($ or $\lambda)$ is $\ell_{U} / f_{U}^{\prime}$ (or defect concentration) always gives a concave curve with a 
negative slope. This condition is general and, indeed, is characteristic of all curves of $\lambda$ as a function of anything (temperature lattice defects due to irradiation, interstitials, porosity, residue compourds) that influences the phonon mean free path.

\section{COMPARISON WITH EXPEKIMENTS}

\section{Defect and Impurity Effects}

Data for testing the ideas presented here are relatively scarce, because the requirement of $L_{a}$ (i.e., ${ }_{B}$ ) constancy makes it mandatory that changes imposed upon a test specimen to alter its thermal conductivity be made either on a single specimen or on several specimens whose constant $L_{a}$ value has been verified experimentally. Furthermore, for anisotropic systems (and graphite certainly fits in that category) evidence that the preferred orientation has remained constant during the doping or structural alteration is required, because orientation changes can cause thermal conductivity changes that can effectively mask the effects being investigated. Although reported measurements on similar "grades" or "batches" of graphites do not normally meet the standards set forth here, some literature data are considered here because the care used in the experiments approaches that required.

A group of experiments performed at this Laboratory on how boron doping affects polycrystalliie graphite properties yielded information on thermal conductivity effects when $L_{a}$ and the degree of preferred orientation were kept constant. These results are shown in Fig. 1, a plot of roomtemperature thermal conductivities of borcn-doped graphites vs boron content. The manufacture, crystallographic parameters, boron dispesition, and effects on other properties of these graphites have been detailed.12 Because the graphite was in the form of extruded rods, $\lambda$ was measured parallel and perpendicular to the extrusion axis to show the effect of orientation. The upper curve in Fig. 1 reflects measuremenis made parallel to the extrusion axis; because the crystallite planes tend to align themselves along the extrusion axis, these $\lambda$ values are higher than those perpendicular to the extrusion axis. The shapes of the curves in Fig. 1 are exactly those predicted from Eq. (15) which is based on the simple phonon-scattering model.

Smith and Rasor ${ }^{17}$ reported on how neutron irradiation and formation of bromine residue compounds affect graphite thermal conductivities. Although $\mathrm{L}_{\mathrm{a}}$ measurements and pertinent crystallographic information were not included in their work, they were careful to ensure that the results

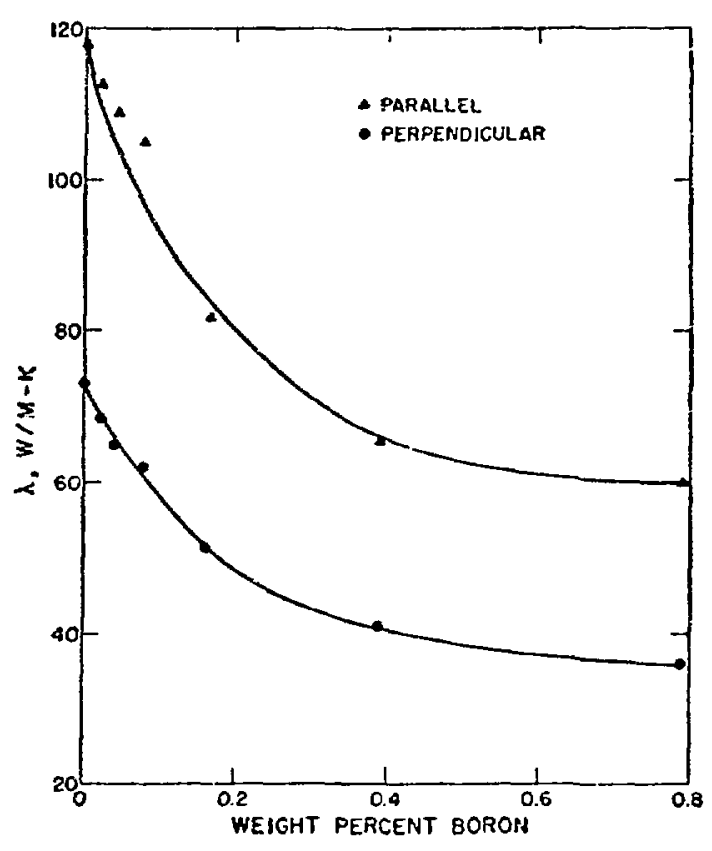

Fig. 1.

Effect of boron on the room temperature thermal conductivity of polycrystalline graphite. Measurements made parallel and perpendicular to the extrusion axis.

were not unduly influenced by variables other than those intended. These data are of particular interest because the neutron irradiation produces a severely defect-laden lattice whereas the bromine residue compounds have their greatest effect on the character of the crystallite boundaries, thus permitting more or less independent consideration of $\ell_{U}$ and ${ }_{B}$ effects. Smith and Rasor presented their data as plots of $\lambda$ vs $\mathrm{T}$ with total neutron exposure or bromine content as an independent parameter. To show the effects pertinent to this work, some of their data are cross-plotted in Fig. 2 to show how integrated neutron flux affects the thermal conductivity (all measurements made at $100 \mathrm{~K}$ ) of $\mathrm{SA}-25$ graphite. Recalling that $\lambda$ varies as $/ / /$ in the phonon-scattering model and $f_{U} / /_{U}^{\prime}$ is proportional to total radiation exposure, we see that the expected concave curve of negative slope is generated when $\lambda$ is plotted vs integrated flux in Fig. 2. These conclusions unply equally well to all of Smith and Rasor's daca on neutron-irradiated graphites and to more rece it results. 17

Graphite and bromine residue compounds are made by allowing bromine to react with graphite to form unstable lamellar compounds and then heating 


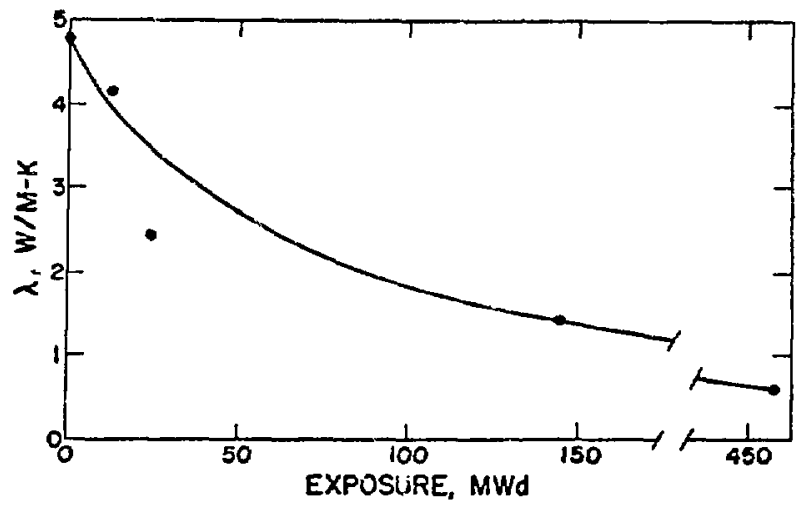

Fig. 2.

Effect of neutron irradiation on thermal conductivity (measured at $100 \mathrm{~K}$ ) of $S A-25$ graphite. One $M W$-day equals $10^{7}$ nvt neutrons with energy greater than $0.5 \mathrm{MeV} .16$

the system and allowing the compounds to decompose. At all but extremely high temperatures $\left(>2000^{\circ} \mathrm{C}\right.$ ), some of the bromine remains behind upon decomposition. Essentially all the remaining bromine resides in the crystallite boundaries and other discontinuities, thus making for a system in which only boundary scattering should be affected, neglecting electron processes. For the AGOT-KC graphite they used, Smith and Rasor estimated that a little more than 1 at. $\%$ of bromine was the most that could be accomodated without damaging the graphite crystallites. Bromine's effect on the thermal conductivity, at $100 \mathrm{~K}$, of AGOT-KC grapiite below this limiting concentration is shown in Fig. 3. Once again, the typical curve shape to be expected from the relation

$$
\frac{l^{\prime}}{l}-\frac{\frac{1+\ell_{B}}{l_{U}}}{\frac{l_{B}}{l_{B}^{\prime}}+\frac{l_{B}}{l_{U}}}
$$

is observed. In this instance, however, note that $t_{\mathrm{B}}$ is not actually changed in dimension; instead, it is the scattering efficiency of the crystallite boundary which has changed. This concept in the same context was discussed by Jamieson and Mrozowski ${ }^{3}$ who used a multiplicative factor, $\gamma_{B}$, to account for the phonon-scattering probability at the boundary. In their notation, $\gamma_{B} L_{B}$ would be equivalent to the ${ }_{B}$ used here and changes in scattering efficiency would be reflected in changed values of $\gamma_{B}$.

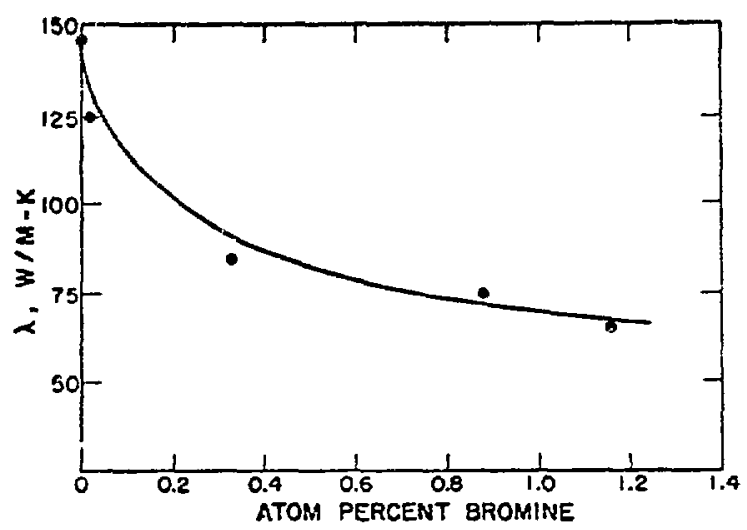

Fig. 3.

Effect of bromine content on thermal conductivity (measured at $100 \mathrm{~K}$ ) of $A G O T-K C$ gruphite. ${ }^{16}$

\section{High and Low Temperature Effects}

At well below room temperature, $t_{\mathrm{V}}$ becomes large enough that $t=t_{\mathrm{B}}$ in extreme cases and where single crystals are used $\delta_{B}$, and thus $\lambda$, is determined by the specimen size. ${ }^{18}$ With decreasing $T, 1 / / /_{B} \gg 1$ and $\ell_{0} / h_{B} \gg \ell_{U} / l_{U}^{\prime}$ and Eq. (13) becomes

$$
\frac{\ell^{\prime}}{\ell}-\frac{1+\frac{\ell_{U}}{\ell_{B}}}{\frac{l_{U}}{l_{U}^{f}}+\frac{\ell_{U}}{l_{B}}}+1 .
$$

Thus, one would expect thermal conductivity curves for materials that are essentially identical, except for different defect concentrations, to converge with decreasing temperature, assuming of course that the maximum temperature considered is low enough so that $f_{\mathrm{U}} / \hat{B}_{\mathrm{B}}$ is either greater than or not too different from unity. Again, data for testing these ideas are scarce because of the required constant $/_{B}$ and orientation. However, Powell and Wagner's work 15 seems to fit this category and some of Smith and Rasor's data ${ }^{16}$ also can be considered. Powell and Wagner used three specimens of commercially available polycrystalline graphites and measured $\lambda$ from 20 to about $100 \mathrm{~K}$. They then irradiated the samples with neutrons and remeasured the thermal conductivity over the same temperature rante. At $100 \mathrm{~K}$, the conductivity was only about $15 \%$ of its preirradiation value; however, comparison of the irradiated specimens' $\lambda$ vs $T$ curves with those plotted before irradiation showed a convergence with decreasing temperature as Eq. (17) predicts. Smith 
and Rasor's plots of $\lambda$ vs $T$ from 20 to about $300 \mathrm{H}$ showed a similar behavior, in that increased neutron exposures led to greater differences in $\lambda$ near room temperature and the therrnal conductivities tended to converge with decreasing temperature. The same is true of their results with the graphite and bromine residue compounds. Thus the low temperature behavior analysis based on the phonon-scattering model seems reasonably well substantiated by experimental evidence, despite anticipation that, by analogy with the electrical properties from which Mathiessen's rule is derived, there would be some residual thermal resistivity at $0 \mathrm{~K}$.

Above room temperature, the $\mathrm{U}$ processes become increasingly important. Generally $f_{\mathrm{C}}$ is proportional to $1 / \mathrm{T}, 19,20$ and $f_{\mathrm{H}} / /_{\mathrm{B}}$ decreases accordingly, whereas the ratio $f_{L} / f_{U}^{\prime}$ should remain fairly constant since both should go as $1 / \bar{\gamma}$ at high temperatures. Then Eq. (13) becomes

$$
\frac{l^{\prime}}{l}=\frac{1+\frac{l_{U}}{l_{B}}}{\frac{l_{U}}{l_{D}^{\prime}}+\frac{l_{U}}{l_{B}}}=\frac{l_{U}^{\prime}}{l_{U}} .
$$

Thus one would expect that $\lambda_{j}^{\prime}$ and $\lambda$, again equating $\lambda$ with $/$ of Eq. (11), plotted vs temperature for graphites that differ only in defect concent ration (j) would show no tendency to converge even at the highest temperatures and would yield a series of curves. This postulate is exceedingly difficult to verify because of the scarcity of germane data. Wagner and Dickinson ${ }^{12}$ published curves of $\lambda$ vs $T$ from about -100 to $2500^{\circ} \mathrm{C}$ for graphites containing small amounts of boron. Although these curves lie in the expected order ( $\lambda_{j}^{\prime}$ decreases with increasing boron content as shown in Fig. 1) and there is no evidence to contradict Eq. (18), it is unfortunate that above $1500^{\circ} \mathrm{C}$ the relative errors in the radial heat flow measurement 21 exceed the expected $\lambda_{j}^{\prime}$ differences between the curves. As no other data that fit the conditions on $\lambda_{B}$ etc. are known, the high temperature analysis must be considered to lack firm experimental backing.

\section{Miscellaneous Observations}

It is of interest to consider the relative values of $f_{U}$ and $\beta_{3}$. This may be done by evaluating Eq. (12) at the point where the thermal conductivity (plotted vs $\mathrm{T})$ goes through a maximum, $\mathrm{d} \lambda / \mathrm{d} T=0$, that for graphite is normally just above room temperature. If Eq. (12) is solved for / and differentiated with respect in temperature, allowing $d / / d T=0$,
$\ell_{U}^{2} \frac{\partial \ell_{B}}{\partial T}+\ell_{B}{ }^{2} \frac{\partial \ell_{U}}{\partial T}=0$.

Near ihe conductivity inaximum, none of the above terms is zero and when

$$
\begin{aligned}
& \frac{\partial L_{B}}{\partial T}=-\frac{\partial \ell_{v}}{\partial T} . \\
& \mathscr{L}_{U}=\ell_{B} .
\end{aligned}
$$

The above conclusion that at maximum thermal conductivity the boundary scattering is equal to the Umklapp scattering has appeared in the literature 22,23 and is hardly startling. However, it is an interesting result in view of the assumption thit there is no electronic contribution to the therna. conductivity and of the extremely simple mechanism implied by considering only two in-plane scattering processes.

Ivanov 24 has suggested that for porous materials (graphite porosity varies from near 0 to 50\%) the resultant mear free path should be formulated as in Eq. (6)

$$
\frac{1}{l}=\frac{1}{l_{U}}+\frac{1}{l_{B}}+\frac{J}{l_{\text {pores }}} .
$$

where pores is a distance between pores. This suggestion was shown to be valid by the work of Wagner, O'Rourke, and Armstrong, ${ }^{25}$ in which pore concentration was the experimental variable of interest. They used 15 graphite samples with $15-32 \%$ porosities. The crystallite heights, lattice spacing, anisotropies, and pore sizes and ahapes were sensibly constant, and the primary variable was the number of pores. For these graphites, Ivanov's relation im. plies that

$$
\begin{aligned}
\lambda & \approx \frac{1}{l} \approx \frac{1}{l_{\text {pores }}} \approx \frac{1}{\text { number of pores }} \\
& \approx \frac{1}{\text { porosity }} .
\end{aligned}
$$

The data were plotted as $1 / \lambda$ vs porosity and fitted a atraight line. This is significant because density corrections to thermal conductivity in graphite have keen difficult to apply, probably because conduction is associated with a scattering phenomenon rather than a simple Maxwellian area correction. 


\section{SUMMARY}

The basic phonon-scattering formulation, Eq. (5), has been shown to be applicable for pyrolytic and polycrystalline graphites in various stages of imperfection and at various temperatures, with no alteration of the original premise. Although temperature effects seem to be reasonably well rationalized in this work, phonon-scattering efficiencies at the crystallite boundaries must be better defined. However, above all, better experiments with carefully controlled or identified structural parameters such as crystallite anisotropies, lattice spacings, and crystallite sizes are needed before any theory of heat conduction in graphite can be shown to correspond to reality.

The implications for more immediate applications are more obvious and concern the formulation for the overall thermal conductivity or multiphase systems. Past custom has been to express thermal conductivities of multiphase systems as combinations of the form

$$
\lambda=\sum_{1=1}^{n} a_{i} \lambda_{i}
$$

or

$$
\lambda^{-1}=\sum_{1=1}^{n} a_{i}\left(\lambda_{1}\right)^{-1} .
$$

The reasons for favoring one form more than the other have never been defined clearly, so both types of expressions have appeared in the literature. Taking graphite as a limiting case of a multiphase system (the pore structure being the second phase), it appears that the second type of expression is quite reasonable, as this work shows. Furthermore, since interfacial boundaries in a multiphase system will act as phonon scatterers, there is reason to use the expression

$$
\frac{1}{\lambda}-\sum_{1=1}^{n} \frac{a_{1}}{\lambda_{1}}
$$

for systems in which phonon propagation plays a major role in heat conduction.

\section{REFERENCES}

1. C. N. Hooker, A. R. Ubbelohde, F. R. S., and D. A. Young, "Anisotropy of Thermal Conductance in Near-Ideal Graphite," Proc. Roy. Soc. (London) A284, 17 (1965).

2. J. E. Hove and A. W. Smith. "Interpretation of Low-Temperature Thermal Conductivity of Grephite," Phys. Rev. 104. 892-900 (1956).

3. C. P. Jamieson and S. Mrozowski, "Thermal Conductivities of Polycrystalline Carbons and Graphites," Proc. Conf. Carbon, Buffalo, New York, 1956 (Waverly Press, Baltimore, 1956), p. 155.

4. Josef Kaspar, "Thermal Propagation in Carbons and Graphites at Very High Temperatures," US Air Force report SSD-TR-67-56 (1967).

5. B. T. Kelly, "The Effect of Defects on the Basal Plane Thermal Conductivity of a Graphite Crystal," Carbon 5, 247-260 (1967).

6. P. G. Klemens, "Thermal Conductivity and Lattice Vibrational Modes," Solid State Phys. 1. 198 (1958).

7. R. Taylor, "The Thermal Conductivity of Pyrolytic Graphite," Phil. Mag. 13, 157 (1966).

8. R. Taylor, B. T. Kelley, and K. E. Gilchrist, "The Thermal Conductivity of Fast Neutron Irradiated Graphite," United Kingdom Atomic Energy Authority report TRG Report 1602(c) (1968).

9. P. G. Klemens, talk given at 4 th Conf. Thermal Conductivity, San Francisco, California, 1964.

10. I. B. Mason and K. H. Knibbs, "The Thermal Conductivity of Artificial Graphites and its Relationship to Eiectrical Resistivity," United Kingdom Atomic Energy Authority report AERER3973 (1962).

11. R. Taylor, K. E. Gilchrist, and L. J. Poston," Thermal Conductivity of Polycrystalline Graphite," Carbon 6, 537-544 (1968).

12. P. Wagner and J. M. Dickinson, "Ambient and High Temperature Experiments on Boron-Doped Polycrystalline Graphites," Carbon 8, 313-320 (1970). 
13. J. M. Ziman, Electrons and Phonons (Cxford University Press, London, 1967), pp. 286, 309.

14. B. T. Kelly, "The Thermal Conductivity of Graphite Parallel to the Basal Planes and the Velocity of Phonons in the "Out-of-Plane' Acoustic Mode," Phil. Mag. 15, 1005 (1967).

15. Robert L. Powell and Paul Wagner, "Irradiation Effects on Low 'Terr. perature Thermal and Electrical Conductivities of Two Graphites," Carbon 8 , 690-692 (1970).

16. Alan W. Smith and Ned S. Rasor, "Observed Dependence of the Low-Temperature Thermal and Electrical Conductivity of Graphite on Temperature, Type, Neutron Irradiation, and Bromination," Phys. Rev, 104, 885 (1956).

17. R. D. Reiswig, IP. Wagner, C. M. Hollabaugh, R. W. White, J. A. O'Rourke, K. V. Davidson, and D. H. Schell, "Behavior of LASL-Made Graphite, $\mathrm{ZrC}$, and $\mathrm{ZrC}$-Containing Coated Particles in Irradiation Tests HT-28 and HT-29," Los Alamos Scientific Laboratory report LA-6211 (1976).

18. H. B. G. Casimir. "Note on the Conduction $o^{\text {f }}$ Heat in Crustals," Physica E, 495 (1938).
19. R. Peierls, "Zur kinetischen Theorie der Wärmeleitung in ńristallen," Ann. Physik. 3, 1055 (1929).

20. G. Leibfried and E. Schlomann, "Warmeleitung in elektrisch isolierenden Kristallen," Nachr. Ak\&z. Wiss. Goettingen IIa, 71 (1954).

21. P. Wagner and L. B. Dauelsberg, "The Thermal Conductivity of ZTA Graphite," Carbon 5, 271 (1967).

22. R. Berman, "The Thermal Conductivity of Dielectric Solids at Low Temperatures," Adv. Phys. 2, 103 (1953).

23. J. G. Castle, "Heat Conduction in Carbon Materials," Proc. Conf. Carbon, Buffalo, New York, 1955 (Waverly Press, Baltimore, 1956), p. 13.

24. B. N. Ivanov, "Theory of Transfer Phenomena in Crystals with Pores. Scattering of Electrons and Phonons by Pores," Teplofiz. Vys. Temp. 4, 875 (1966).

25. P. Wagner, J. A. O'Rourke, and P. E. Armstrong, "Porosity Effects in Polycryetalline Graphite," J. Am. Ceram. Soc. 55, 214 (1972). 